\title{
Evolutionary Design of an X-Band Antenna for NASA's Space Technology 5 Mission
}

\author{
Jason D. Lohn \\ Computational Sciences Division \\ NASA Ames Research Center \\ Moffett Field, CA 94035-1000 \\ jlohn@email. arc.nasa.gov \\ Gregory S. Hornby \\ QSS Group, Inc. \\ NASA Ames Research Center \\ Moffett Field, CA 94035-1000 \\ hornby@email . arc.nasa.gov \\ Adán Rodríguez-Arroyo \\ Microwave Systems Branch \\ NASA Goddard Space Flight Center \\ Greenbelt, MD 20771 \\ Adan.Rodriguez-1@nasa.gov
}

\author{
Derek S. Linden \\ Linden Innovation Research \\ P.O. Box 1601 \\ Ashburn, VA 20146-1601 \\ dlinden@lindenir.com \\ William F. Kraus \\ QSS Group, Inc. \\ NASA Ames Research Center \\ Moffett Field, CA 94035-1000 \\ bkraus@email .arc.nasa.gov \\ Stephen E. Seufert \\ Microwave Systems Branch \\ NASA Goddard Space Flight Center \\ Greenbelt, MD 20771 \\ Stephen.E.Seufert@nasa.gov
}

\begin{abstract}
We present an evolved $X$-band antenna design and fight prototype currently on schedule to be deployed on NASA's Space Technology 5 spacecraft in 2004. The mission consists of three small satellites that will take science measurements in Earth's magnetosphere. The antenna was evolved to meet a challenging set of mission requirements, most notably the combination of wide beamwidth for a circularly-polarized wave and wide bandwidth. Two genetic algorithms were used: one allowed branching in the antenna arms and the other did not. The highest performance antennas from both algorithms were fabricated and tested. $A$ handdesigned antenna was produced by the contractor responsible for the design and build of the mission antennas. The hand-designed antenna is a quadrifilar helix, and we present performance data for comparison to the evolved antennas. As of this writing, one of our evolved antenna prototypes is undergoing fight qualification testing. If successful, the resulting antenna would represent the first evolved hardware in space, and the first deployed evolved antenna.
\end{abstract}

\section{Introduction}

The spectrum of antenna designs for applications in communication, radar, and remote sensing systems is vast, and there is an increasing need for highperformance, customized antennas. Current methods of designing and optimizing antennas by hand are time and labor intensive, limit complexity, increase the time and cost expended, and require that antenna engineers have significant knowledge of the universe of antenna designs. Designers are aided by local optimization techniques, however an initial guess that is close to the final design is required. Evolutionary algorithms such as the genetic algorithm (GA) $[8,7]$, do not require an initial guess and the amount of design parameters that an engineer must provide can be very minimal. Evolutionary algorithms show promise because they have proven ability to search large, unknown design spaces.

Researchers have been investigating evolutionary antenna design and optimization since the early 1990s (e.g., $[16,9,3]$ ), and the field has grown in recent years as computer speed has increased and electromagnetics simulators have improved. Many antenna types have been investigated, including wire antennas [11], an- 
tenna arrays [10], and quadrifilar helical antennas [15]. In addition, the ability to evolve design antennas insitu [13], that is, taking into account the effects of surrounding structures, opens new design possibilities. Such an approach is very difficult for antenna designers due to the complexity of electromagnetic interactions, yet easy to integrate into evolutionary techniques.

Below we describe an evolutionary algorithm approach to a challenging antenna design problem on NASA's Space Technology 5 (ST5) mission [18]. ST5 is one of NASA's New Millennium Program missions that will launch multiple miniature spacecraft to test innovative concepts and technologies in the harsh environment of space. During flight validation of its technologies, ST5 may measure the effect of solar activity on the Earth's magnetosphere. ST5's objective is to demonstrate and flight qualify innovative technologies and concepts for application to future space missions. Images showing the ST5 spacecraft are seen in Figure 1. The mission duration is planned for three months.
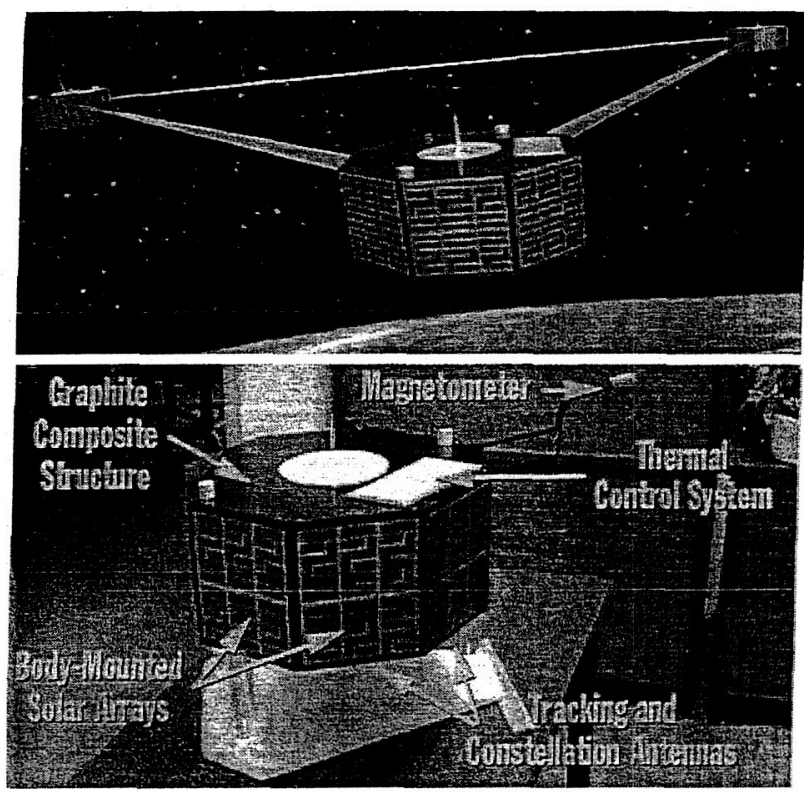

Figure 1. Artist's depiction of the ST5 mission (top), satellite mockup (bottom). Each satellite will have two antennas, centered on the top and bottom of each spacecraft.

\section{ST5 Mission Antenna Requirements}

The three ST5 spacecraft will orbit at close separations in a highly elliptical geosynchronous transfer orbit

\begin{tabular}{||l|l||}
\hline Property & Specification \\
\hline \hline Transmit Frequency & $8470 \mathrm{MHz}$ \\
\hline Receive Frequency & $7209.125 \mathrm{MHz}$ \\
\hline VSWR & $\begin{array}{l}<1.2: 1 \text { at Transmit Freq } \\
<1.5: 1 \text { at Receive Freq }\end{array}$ \\
& $\geq 0 \mathrm{dBic}, 40^{\circ} \leq \theta \leq 80^{\circ}$, \\
\hline Gain Pattern & $0^{\circ} \leq \phi \leq 360^{\circ}$ \\
\hline Input Impedance & $50 \Omega$ \\
\hline Diameter & $<15.24 \mathrm{~cm}$ \\
\hline Height & $<15.24 \mathrm{~cm}$ \\
\hline Antenna Mass & $<165 \mathrm{~g}$ \\
\hline
\end{tabular}

Table 1. Key ST5 Antenna Requirements

approximately $35,000 \mathrm{~km}$ above Earth and will communicate with a 34 meter ground-based dish antenna. The key ST5 mission antenna requirements are summarized in Table 1. The combination of wide beamwidth for a circularly-polarized wave and wide bandwidth make for a challenging design problem. In terms of simulation challenges, because the diameter of the spacecraft is $54.2 \mathrm{~cm}$, the spacecraft is $13-15$ wavelengths across which makes antenna simulation computationally intensive. For that reason, an infinite ground plane approximation or smaller finite ground plane is typically used in modeling and design.

In addition to the requirements in Table 1 , an additional "desired" specification was issued for the field pattern. Because of the spacecraft's relative orientation to the Earth, high gain in the field pattern was desired at low elevation angles. Specifically, across $0^{\circ} \leq \phi \leq 360^{\circ}$, gain was desired to meet: $0 \mathrm{dBic}$ for $\theta=40^{\circ}, 2 \mathrm{dBic}$ for $\theta=80^{\circ}$, and $4 \mathrm{dBic}$ for $\theta=90^{\circ}$.

Based on the above requirements, the Electromagnetic Systems Branch, a component of the Physical Science Laboratory (PSL) at New Mexico State University, was awarded the contract to design and build the antennas for the ST5 mission. The PSL proceeded using conventional design practices, relying on human expertise and antenna computer-aided design software to produce a compliant design and prototype. In parallel, our group worked on the evolutionary design and fabrication described in this paper and proceeded independently of the PSL's work.

ST5 mission managers were willing to accept antenna performance that aligned closer to the "desired" field pattern specifications noted above, and the PSL team designed a quadrifilar helical (QFH) antenna to meet these parameters (see Figure 2). Thus, the QFH antenna, while it does not strictly meet the field pattern requirements as shown in Table 1, it achieves high 
performance and is acceptable to the mission managers.

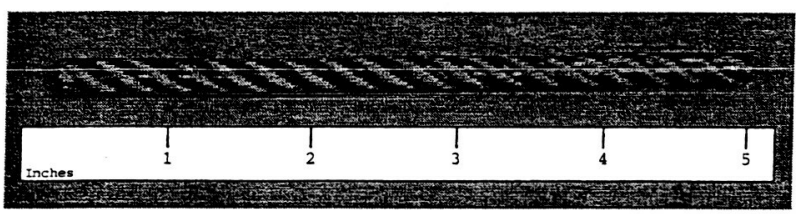

(a)

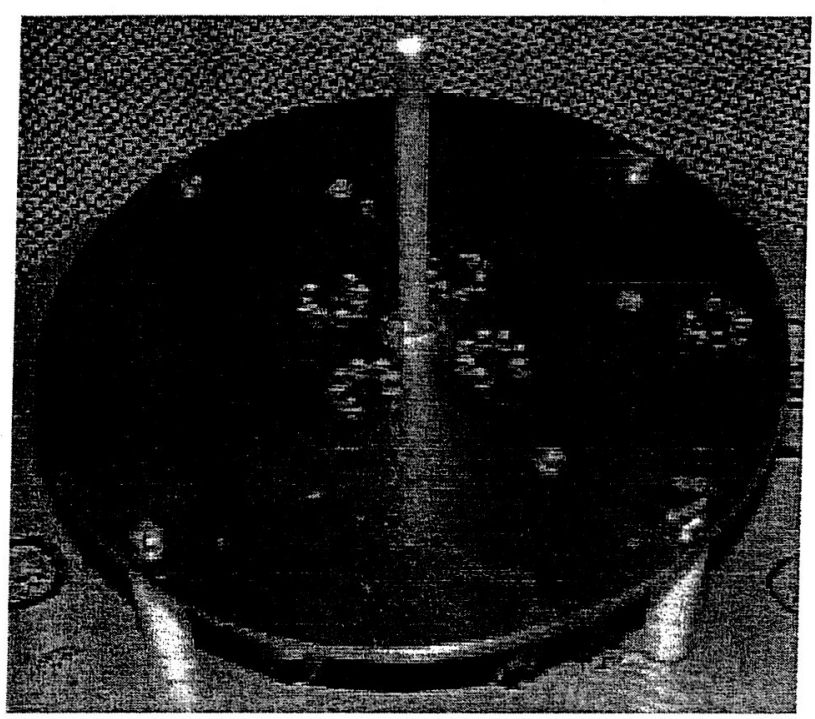

(b)

Figure 2. Quadrifilar helical (QHF) antenna designed and built by the Physical Science Laboratory (PSL) at New Mexico State University: (a) Radiator; (b) Radiator mounted on ground plane.

\section{Evolved Antenna Design}

From past experience in designing wire antennas [12], we decided to constrain our evolutionary design to a monopole wire antenna with four identical arms, each arm rotated $90^{\circ}$ from its neighbors. The GA thus evolves genotypes that specify the design for one arm, and builds the complete antenna using four copies of the evolved arm.

In the remainder of this section we describe the two genetic algorithms used. One GA allows for branching in the arms, and the other is non-branching.

\subsection{Branching GA}

The $\overline{\mathrm{GA}}$ in this section allows for branching in the antenna arms. Rather than using linear sequences of bits or real-values as is traditionally done, here we use a tree-structured representation which naturally represents branching in the antenna arms.

\section{Representation}

A LOGO-like antenna constructing programming language was devised. These are based on those for creating line drawings with a LOGO-style turtle [1]. Each node in the tree-structured representation is an antenna-construction command and an antenna is created by starting from the root of the tree and executing the commands at each node in the tree, starting with the root node. In constructing an antenna the current state (location and orientation) is maintained and commands add wires or change the current state. The commands are as follows:

- forward(length, radius) - add a wire with the given length and radius extending from the current location and then change the current state location to the end of the new wire.

- rotate-x(angle) - change the orientation by rotating it the specified amount (in radians) about the $\mathrm{x}$-axis.

- rotate-y(angle) - change the orientation by rotating it the specified amount (in radians) about the $\mathrm{y}$-axis.

- rotate-z(angle) - change the orientation by rotating it the specified amount (in radians) about the $z$-axis.

An antenna design is created by starting with an initial feedwire and adding wires. For the ST5 mission the initial feed wire starts at the origin and has a length of $0.4 \mathrm{~cm}$ along the $\mathrm{Z}$-axis. That is, the design starts with the single feedwire from $(0.0,0.0,0.0)$ to $(0.0$, $0.0,0.4)$ and the current construction state (location and orientation) is the next wire will be started from location $(0.0,0.0,0.4)$ and the orientation is along the positive Z-axis.

To produce antennas that are four-way symmetric about the $\mathrm{z}$-axis, the construction process is restricted to producing antenna wires that are fully contained in the positive XY quadrant and then after construction is complete this arm is copied three times and these copies are placed in each of the other quadrants through rotations of $90^{\circ} / 180^{\circ} / 270^{\circ}$. For example, in executing the program rotate $z(0.523598776)$ forward $(1.0,0.032)$, the rotate $\mathrm{z}()$ command causes the the current orientation to rotate 0.523598776 radians $\left(30^{\circ}\right)$ about the $Z$ axis. The forward() command adds a wire of length 
$1.0 \mathrm{~cm}$ and radius $0.032 \mathrm{~cm}$ in the current forward direction. This wire is then copied into each of the other three $X Y$ quadrants. The resulting antenna is shown in Figure 3(a).

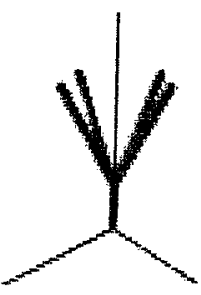

(a)

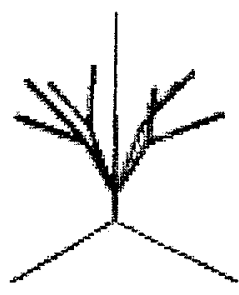

(b)
Figure 3. Example antennas: (a) non-branching arms; (b) branching arms.

Branches in the representation cause a branch in the flow of execution and create different branches in the constructed antenna. The following is an encoding of an antenna with branching in the arms, here brackets used to separate the subtrees:

rotate $-z(0.523598776)$ [ forward $(1.0,0.032)$ [

rotate $-z(0.523598776)$ [ forward $(1.0,0.032)]$

rotate $-x(0.523598776)$ [ forward $(1.0,0.032)]$

] ]

This antenna is shown in Figure 3(b).

To take into account imprecision in manufacturing an antenna, antenna designs are evaluated multiple times, each time with a small random perturbation applied to joint angles and wire radii. The overall fitness of an antenna is the worst score of these evaluations. In this way, the fitness score assigned to an antenna design is a conservative estimate of how well it will perform if it were to be constructed. An additional side-effect of this is that antennas evolved with this manufacturing noise tend to perform well across a broader range of frequencies than do antennas evolved without this noise.

\section{Fitness Function}

The fitness function used to evaluate antennas is a function of the voltage standing wave ratio (VSWR) and gain values on the transmit and receive frequencies. VSWR is a way to quantify reflected-wave interference, and thus the amount of impedance mismatch at the junction. VSWR is the ratio between the highest voltage and the lowest voltage in the signal envelope along a transmission line. The VSWR component of the fitness function is constructed to put strong pressure to evolving antennas with receive and transmit
VSWR values below the required amounts of 1.2 and 1.5 , reduced pressure at a value below these requirements (1.15 and 1.25) and then no pressure to go below 1.1:

$$
\begin{aligned}
v_{r} & =\text { VSWR at receive frequency } \\
v_{r}^{\prime} & = \begin{cases}v_{r}+2.0\left(v_{r}-1.25\right) & \text { if } v_{r}>1.25 \\
v_{r} & \text { if } 1.25>v_{r}>1.1 \\
1.1 & \text { if } v_{r}<1.1\end{cases} \\
v_{t} & =\text { VSWR at transmit frequency } \\
v_{t}^{\prime} & = \begin{cases}v_{t}+2.0\left(v_{t}-1.15\right) & \text { if } v_{t}>1.15 \\
v_{t} & \text { if } 1.15>v_{t}>1.1 \\
1.1 & \text { if } v_{t}<1.1\end{cases} \\
v s w r & =v_{r}^{\prime} v_{t}^{\prime}
\end{aligned}
$$

The gain component of the fitness function uses the gain (in decibels) in $5^{\circ}$ increments about the angles of interest: from $40^{\circ} \leq \theta \leq 90^{\circ}$ and $0^{\circ} \leq \phi \leq 360^{\circ}$ :

$$
\begin{aligned}
\operatorname{gain}_{i j} & =\text { gain at } \theta=5^{\circ} i, \phi=5^{\circ} j \\
\operatorname{gain}(i, j) & = \begin{cases}0 & \text { if } \text { gain }_{i j}>0.5 \\
0.5-\text { gain }_{i j} & \text { if gain } \\
\text { gij } & <0.5\end{cases} \\
\text { gain } & =1+0.1 \sum_{i=8}^{i<19} \sum_{j=0}^{j=72} \operatorname{gain}(i, j)
\end{aligned}
$$

While the actual minimum required gain value is 0 $\mathrm{dBic}$ for $40^{\circ} \leq \theta \leq 80^{\circ}$, and desired gain values are 2 $\mathrm{dBic}$ for $\theta \geq 80^{\circ}$ and $4 \mathrm{dBic}$ for $\theta=90^{\circ}$ only a single target gain of $0.5 \mathrm{dBic}$ is used here. This provides some headroom to account for errors in simulation over the minimum of $0 \mathrm{dBic}$ and does not attempt to meet desired gain values. Since achieving gain values greater than $0 \mathrm{dBic}$ is the main part of the require specifications, the third component of the fitness function rewards antenna designs for having sample points with gains greater than zero:

$$
\begin{aligned}
\operatorname{outlier}(i, j) & = \begin{cases}0.1 & \text { if } \text { gain }_{i j}<0.01 \\
0 & \text { otherwise }\end{cases} \\
\text { outlier } & =1+\sum_{i=8}^{i<19} \sum_{j=0}^{j=72} \text { outlier }(i, j)
\end{aligned}
$$

These three components are multiplied together to produce the overall fitness score of an antenna design:

$$
F=\text { vswr } \times \text { gain } \times \text { outlier }
$$

The objective of the GA is to produce antenna designs that minimize $F$. 


\subsection{Non-branching GA}

In this GA, the design was constrained to nonbranching arms and the encoding used real numbers. The feed wire for the antenna is not optimized, but is specified by the user. The size constraints used, an example of an evolved arm, and the resulting antenna are shown in Figure 4.

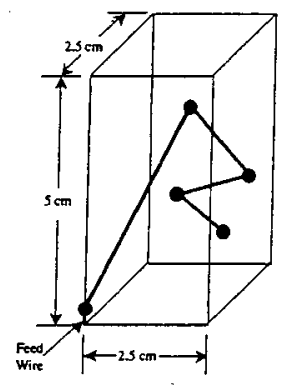

(a)

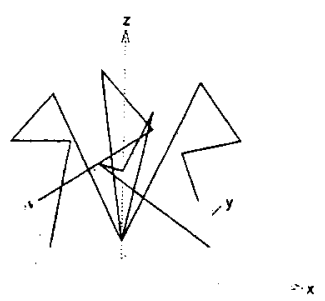

(b)
Figure 4. (a) size constraints and evolved arm; (b) resulting 4-wire antenna after rotations.

\section{Representation}

The design is specified by a set of real-valued scalars, one for each coordinate of each point. Thus, for a foursegment design (shown in Figure 4), 12 parameters are required.

Adewuya's method of mating [2] and Gaussian mutation to evolve effective designs from initial random populations. It has been shown to work extremely well on many different antenna problems $[4,5,14]$.

\section{Fitness Function}

This GA used pattern quality scores at $7.2 \mathrm{GHz}$ and $8.47 \mathrm{GHz}$ in the fitness function. Unlike the previous GA, VSWR was not used in this fitness calculation. To quantify the pattern quality at a single frequency, $P Q_{f}$, the following was used:

$$
\mathrm{PQ}_{f}=\sum_{\substack{0^{\circ}<\phi<360^{\circ} \\ 40^{\circ}<\theta<80^{\circ}}}\left(\text { gain }_{\phi, \theta}-T\right)^{2} \text { if } \text { gain }_{\phi, \theta}<T
$$

where gain $_{\phi, \theta}$ is the gain of the antenna in dBic (righthand polarization) at a particular angle, $T$ is the target gain ( $3 \mathrm{dBic}$ was used in this case), $\phi$ is the azimuth, and $\theta$ is the elevation.

To compute the overall fitness of an antenna design, the pattern quality measures at the transmit and receive frequencies were summed, lower values corresponding to better antennas:

$$
F=\mathrm{PQ}_{7.2}+\mathrm{PQ}_{8.47}
$$

\section{GA Run Setup}

As mentioned earlier, the ST5 spacecraft is $13-15$ wavelengths wide, which makes simulation of the antenna on the full craft very compute intensive. To keep the antenna evaluations fast, an infinite ground plane approximation was used in all runs. This was found to provide sufficient accuracy to achieve several good designs. Designs were then analyzed on a finite ground plane the same shape and size as the top of the ST5 body to determine their effectiveness at meeting requirements in a realistic environment.

The Numerical Electromagnetics Code, Version 4 (NEC4) [6] was used to evaluate all antenna designs.

For the branching GA; a population of 200 individuals and a mutation rate of $50 \%$ was used. For the non-branching GA, a population of 50 individuals was used, $50 \%$ of which is kept from generation to generation. The mutation rate was $1 \%$, with the Gaussian mutation standard deviation of $10 \%$ of the value range. The GA was halted after 100 generations had been completed, the GA's best score was stagnant for 40 generations, or GA's average score was stagnant for 10 generations.

\section{Evolved Antenna Results}

As the goal of our work was to produce requirements-compliant antennas for ST5, no attempt was made to compare the algorithms, either to each other, nor to other search techniques. Thus statistical sampling across multiple runs was not performed.

The two best antennas found, one from each of the GAs described above, were fabricated and tested. The antenna named ST5-3-10 was produced by the GA that allowed branching, and the antenna named ST5-4W03 was produced by the other GA. Photographs of the prototyped antennas are shown in Figure 5. Due to space limitations, only performance data from antenna ST5-3-10 is presented below.

Evolved antenna ST5-3-10 is $100 \%$ compliant with the mission antenna performance requirements. This was confirmed by testing the prototype antenna in an anechoic test chamber at NASA Goddard Space Flight Center (see Figure 6). The data measured in the test chamber is shown in the plots below.

The genotype of antenna ST5-3-10 is shown in Figure 7. The complexity of this large antenna- 
constructing program as compared to the antenna arm design having one branch, suggests that it is not a minimal description of the design. For example, instead of using the minimal number of rotations to specify relative angles between wires (two) there are sequences of up to a dozen rotation commands.

The VSWR plot measured on the ST5-3-10 prototype is shown in Figure 8. The VSWR values are slightly higher than the requirements specify because of mismatch issues on the test equipment. After proper matching, the VSWR values are predicted to be within specification.

The $7.2 \mathrm{GHz} \mathrm{max} / \mathrm{min}$ gain patterns for both evolved antenna ST5-3-10 and the QHA are shown in Figure 9. The $8.47 \mathrm{GHz} \max / \mathrm{min}$ gain patterns for both antennas are shown in Figure 10. On the plots for antenna ST5-3-10, a box denoting the acceptable performance according to the requirements is shown. Note that the minimum gain falls off steeply below $20^{\circ}$. This is acceptable as those elevations were not required due to the orientation of the spacecraft with respect to Earth. As noted above, the QHA antenna was optimized at the $8.47 \mathrm{GHz}$ frequency to achieve high gain in the vicinity of $75^{\circ}-90^{\circ}$.

\section{Results Analysis}

Antenna ST5-3-10 is a requirements-compliant antenna that was built and tested on an antenna test range. While it is slightly difficult to manufacture with out the aid of automated wire-forming and soldering machines, it has a number of benefits as compared to the conventionally-designed antenna.

First, there are potential power savings. Antenna ST5-3-10 achieves high gain (2-4dB) across a wider range of elevation angles. This allows a broader range of angles over which maximum data throughput can be achieved. This would result in less power being required from the solar array and batteries.

Second, the evolved antenna does not require a matching network nor phasing circuit, removing two steps in design and fabrication of the antenna. A trivial transmission line may be used for the match on the flight antenna, but simulation results suggest that one is not required if small changes to the feedpoint are made.

Third, the evolved antenna has more uniform coverage in that it has a uniform pattern with small ripples in the elevations of greatest interest $\left(40^{\circ}-80^{\circ}\right)$. This allows for reliable performance as elevation angle relative to the ground changes.

Fourth, the evolved antenna had a shorter design cycle. It was estimated that antenna ST5-3-10 took 3

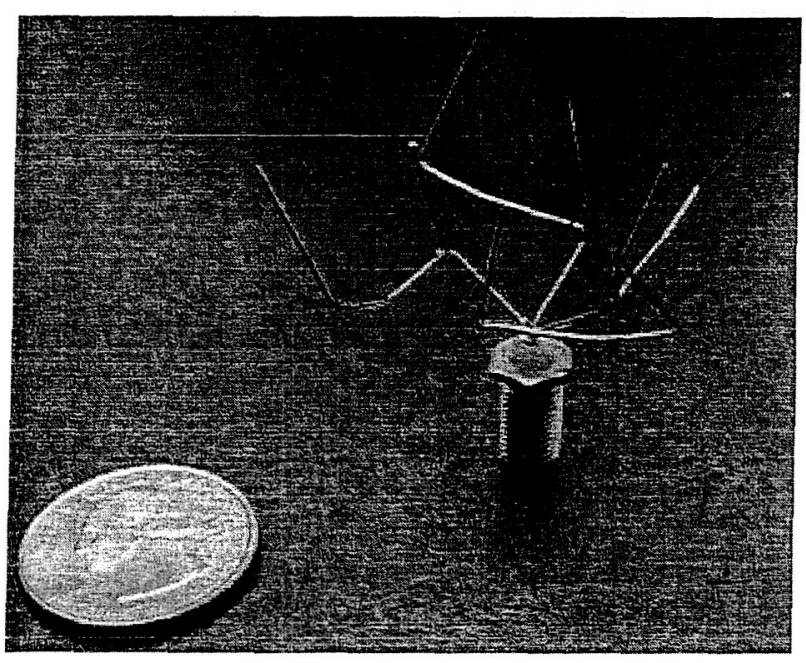

(a)

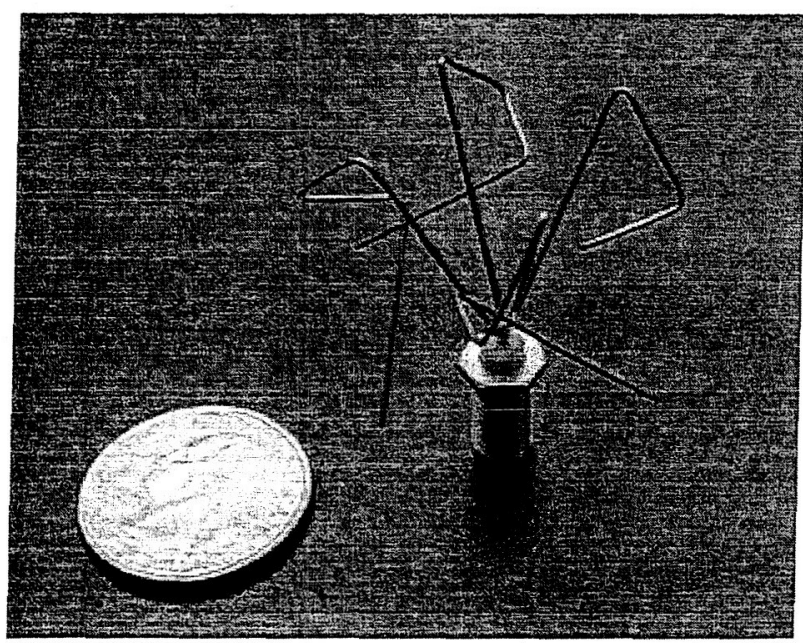

(b)

Figure 5. Photographs of prototype evolved antennas: (a) ST5-3-10; (b) ST5-4W-03 
person-months to design and fabricate the first prototype as compared to 5 person-months for the quadrifilar helical antenna.

\section{Conclusion}

We have evolved and built two X-band antennas for potential use on NASA's upcoming ST5 mission to study the magnetosphere. ST5 antenna requirements, our evolutionary algorithms, and the resulting antennas and performance plots were presented.

Evolved antenna ST5-3-10 was shown to be compliant with respect to the ST5 antenna performance requirements. It has an unusual organic-looking structure, one that expert antenna designers would likely not produce.

If flight qualification testing is successful, antenna ST5-3-10 would represent the first evolved hardware in space, and the first deployed evolved antenna. As the mission's primary goal is to test and validate new technologies for future NASA missions, flying an evolved antenna would fulfill this goal.

\section{Acknowledgments}

The work described in this paper was supported by Mission and Science Measurement Technology, NASA Headquarters, under its Computing, Information, and Communications Technology Program. The work was performed at the Computational Sciences Division, NASA Ames Research Center, Linden Innovation Research, and NASA Goddard Space Flight Center. The support of Ken Perko of Microwave Systems Branch at NASA Goddard and Bruce Blevins of the Physical Science Laboratory at New Mexico State University is gratefully acknowledged.

\section{References}

[1] H. Abelson, Apple LOGO, Peterborough, NH: BYTE/McGraw-Hill, 1982.

[2] A. Adewuya. "New Methods in Genetic Search with Real-valued Chromosomes," Master's Thesis, Mech. Engr. Dept., MIT, 1996.

[3] E.E. Altshuler and D.S. Linden. "Design of a Loaded Monopole Having Hemispherical Coverage Using a Genetic Algorithm." IEEE Trans. Antennas $\mathcal{E}$ Propagation, vol. 45, no. 1, January 1997.

[4] E.E. Altshuler and D.S. Linden. "Wire Antenna Designs using a Genetic Algorithm." IEEE Antenna \& Propagation Society Magazine, vol. 39, pp. 33-43, April 1997.
[5] E.E. Altshuler. "Electrically Small Self-resonant Wire Antennas Optimized using a Genetic Algorithm," IEEE Trans. Antennas Propagat., vol. 50, March 2002, pp. 297-300.

[6] G.J. Burke, A.J. Poggio. "Numerical Electromagnetics Code (NEC)-Method of Moments," Rep. UCID18834, Lawrence Livermore Lab., Jan. 1981.

[7] D.E. Goldberg, Genetic Algorithms in Search, Optimization, and Machine Learning, AddisonWesley, Reading, Mass, 1989.

[8] J.H. Holland, Adaptation in Natural and Artificial Systems, Univ. of Michigan Press, Ann Arbor, 1975.

[9] R.L. Haupt, "An Introduction to Genetic Algorithms for Electromagnetics," IEEE Antennas \& Propagation Mag., vol. 37, April. 1995, pp. 7-15.

[10] R.L. Haupt, "Genetic Algorithm Design of Antenna Arrays," IEEE Aerospace Applications Conf., vol. 1, Feb. 1996, pp. 103-109.

[11] D.S. Linden and E.E. Altshuler. "Automating Wire Antenna Design using Genetic Algorithms," Microwave Journal, Vol. 39, No. 3, March 1996.

[12] D.S. Linden, "Automated Design and Optimization of Wire Antennas using Genetic Algorithms." Ph.D. Thesis, MIT, September 1997.

[13] D.S. Linden. "Wire Antennas Optimized in the Presence of Satellite Structures using Genetic AIgorithms." IEEE Aerospace Conf., April 2000.

[14] D.S. Linden and R.T. MacMillan. "Increasing Genetic Algorithm Efficiency for Wire Antenna Design using Clustering." ACES Special Journal on Genetic Algorithms, 2000.

[15] J.D. Lohn, W.F. Kraus, D.S. Linden, "Evolutionary Optimization of a Quadrifilar Helical Antenna," Proc. of the IEEE Antenna \& Propagation Society Mtg., June 2002, vol. 3, pp. 814-817.

[16] E. Michielssen, J.-M. Sajer, S. Ranjithan, R. Mittra, "Design of Lightweight, Broad-band Microwave Absorbers Using Genetic Algorithms," IEEE Trans. Microwave Theory \& Techniques, vol. 41, no. 6 , June/July 1993, pp. 1024-1031.

[17] Electromagnetic Optimization by Genetic Algorithms, Y. Rahmat-Samii and E. Michielssen (eds.), Wiley, 1999.

[18]. Space -..Technology 5 -Mission, Website: http://nmp.jpl.nasa.gov/st5/ 


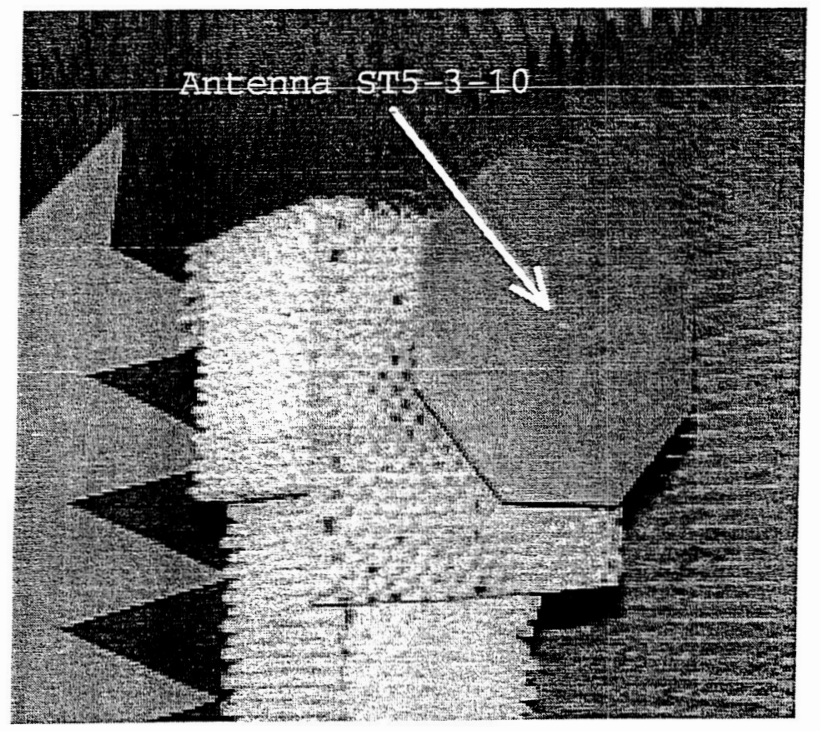

Figure 6. Test chamber setup. Antenna. ST5-3-10 is mounted in the center of the ground plane.

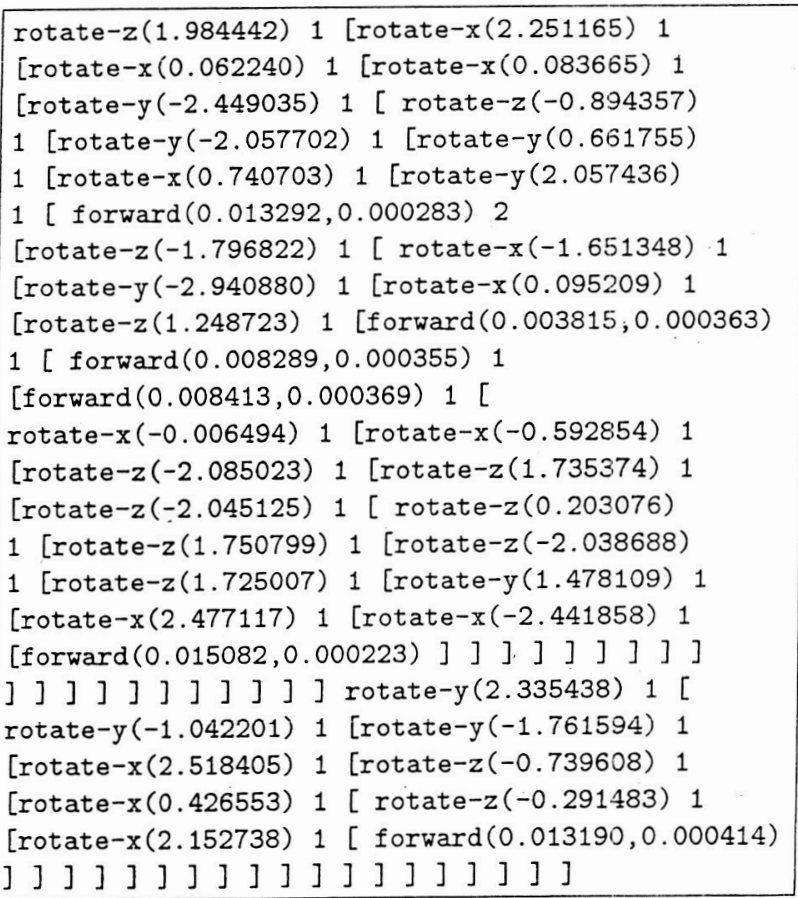

Figure 7. Genotype for evolved antenna ST5-3-10.

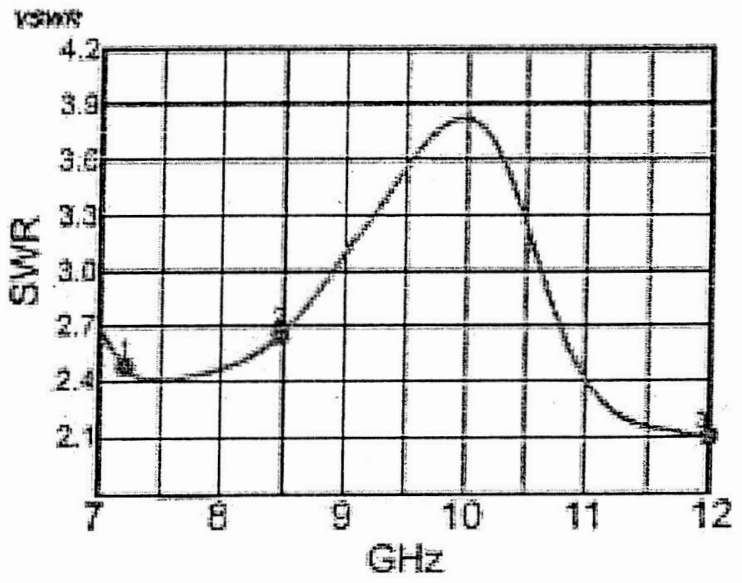

Figure 8. Measured VSWR for antenna ST5-3-10.

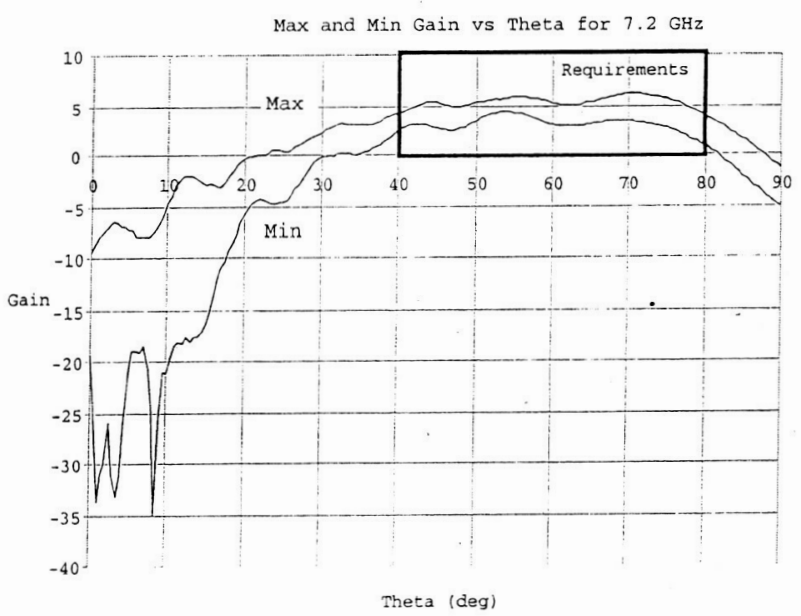

(a)

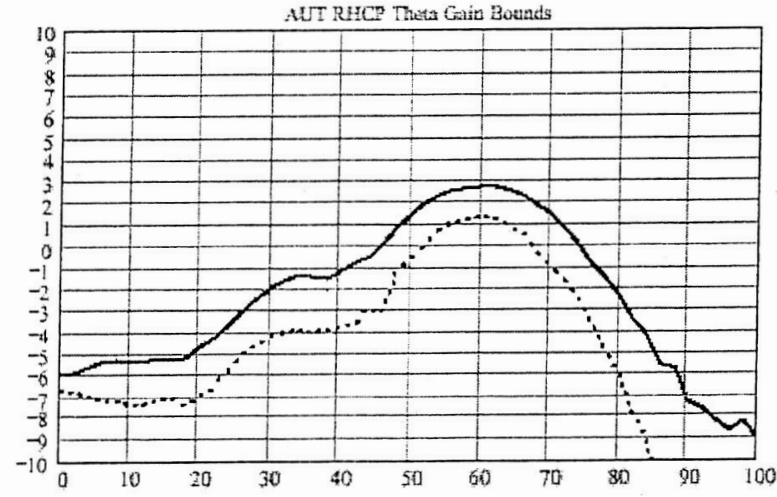

(b)

Figure 9. Maximum and minimum gain at 7.2 $\mathrm{GHz}$ for antennas (a) ST5-3-10; (b) QFH. 


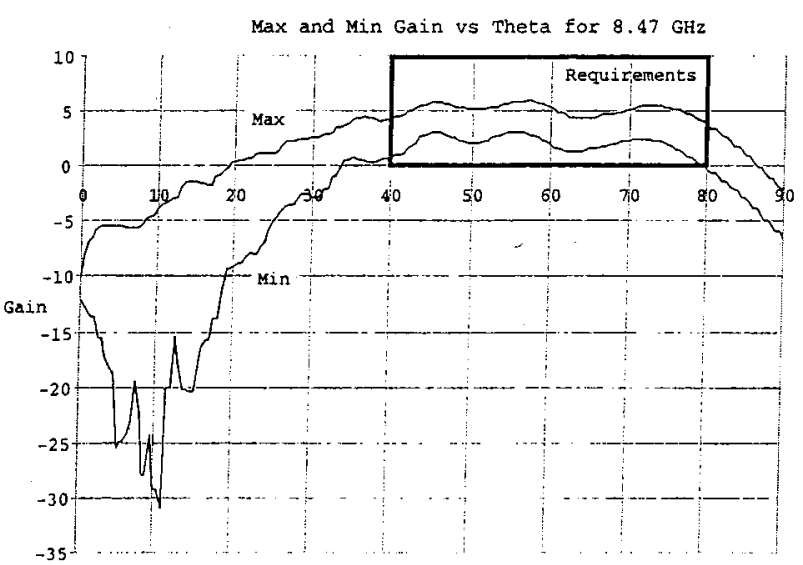

Theta (deg)

(a)

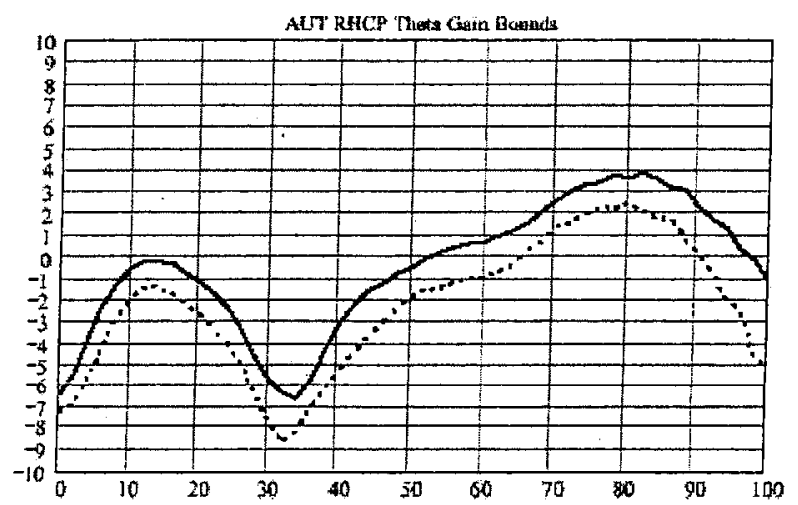

(b)

Figure 10. Maximum and minimum gain at 8.47 $\mathrm{GHz}$ for antennas (a) ST5-3-10; (b) QFH. 